\title{
Aktuelle Befunde über Fischkrankheiten in deutschen Küstengewässern
}

\author{
Heino Mäller
}

\section{Zusammenfassung}

In den 1980er Jahren wurden umtangreiche Bestandsaufnahmen zur Formenvielfalt der Fischkrankheiten sowie der saisonalen und regionalen Fluktuation ihrer Befallsraten in deutschen Küstengewässern angestellt. Sowohl Formenvielfalt als auch viele der Befallsraten scheinen im Wattenmeer und insbesondere in den dortigen Flußmündungen größer zu sein als in Vergleichsgewässern.

Drei "neue" Krankheitsformen werden vorgestellt: die nahrungsinduzierte Maulgranulomatose des Stints, die vermutlich durch Flexibacter-Bakterien verursachte Gelbe Pest des Kabeljaus und ein Fibrom des Steinpickers, in dem lentivirusähnliche Partikel nachgewiesen wurden.

Das Schwergewicht der bisherigen Forschungsvorhaben lag aut dem Sammeln von Felddaten. Mangels experimenteller Arbeitsansätze müssen viele wichtige Fragen zur Bedeutung dieser Krankheiten daher heute noch unbeantwortet bleiben. So bleibt die Vermutung unbewiesen, daß ein Reihe von parasitenbedingten Krankheiten einen entscheidenden Beitrag zur natürlichen Sterblichkeit vor allem von jungen Nutzfïschen im Wattenmeer liefert. Auch das komplizierte Wirkungsgefüge anthropogener und natürlicher Faktoren bei der Entstehung von Fischkrankheiten im Gebiet wird ohne gezielte experimentelle Arbeiten nicht aufzudecken sein.

Das Auftreten von Gelber Pest. Ichthyophonus-Krankheit und lentivirusähnlichen Partikeln in Fischtumoren stellt ein beträchtliches Gefährdungspotential für die deutsche Fischwirtschaft dar. Allgemein ist festzustellen, daß in Deutschland eine große Empfänglichkeit gegenüber Umweltproblemen auf dem Gebiet der Marinen Pathologie besteht. Die nationalen Forschungskapazitäten sind aufgrund anderer, meist kurzfristig angelegter Arbeitsschwerpunkte jedoch nicht ausreichend darauf vorbereitet, diese Probleme auch erfolgreich zu analysieren und zu bewerten.

Als Konsequenz wird gefordert, der bislang vernachlässigten Grundlagenforschung auf dem Gebiet der Marinen Pathologie neben der reinen Datenerhebung auf See künftig eine größere Bedeutung zuzumessen. Das gilt insbesondere für experimentelle und für biochemisch- und mikroskopisch-diagnostische Arbeiten. Künftig auftretende Probleme werden nur dann rechzeitig zu bewerten und zu lösen sein, wenn sich ein ausreichend breit angelegtes und fundiertes Wissenspotential kontinuierlich weiterentwickeln kann.

Findings of investigations into fish diseases in the coastal waters of the North Sea and Baltic Sea (Summary)

In the 1980s, extensive surveys were clone of the fish diseases and their seasonal and regional fluctuations in German coastal waters. Fish diseases and many of their rates of incidence seem to be greater in the Wadden Sea, in particular its estuaries, than in water bodies used for comparison purposes. 
Three "new" diseases are described: hood-induced buccal granulomatosis in smelt: yellow pest in cod, presumably caused by bacteria of the genus Flexibacter; and fibroma in hooknose in which lentivirus-like particles were found.

Until now, research projects have concentrated on data collection in the field. Because insufficient experiments have been carried out, many important questions about the significance of these cliseases still remain unanswered. It is believed that a number of parasite-induced diseases contribute substantially to the natural mortality of commercially interesting fish - especially juveniles - in the Wadden Seal, but this assumption remains unproven. The complex relationship between anthropogenic and natural factors in the genesis of fish diseases in the area will not be understood either if experiments are not carried out.

The occurrence of yellow pest, Ichthyophonus disease and lentivirus-like particles in fish tumours poses a serious potential threat to the German fishing industry. Generally, the German public is very receptive to environmental problems where marine pathology is concerned. But because the emphasis is placed on other, short-term reseach projects. national research bodies are not able to analyze and evaluate such problems successfully.

In addition to datal collection alt sea, greater importance should be attached to pure research in marine pathology, a hitherto neglected field, and particularly to experimental. biochemical and microscopic/diagnostic work. Problems arising in future can only be assessed and solved in time if knowledge is sufficiently broald-based and sound and can continue to increase.

Wer einmal aufmerksam in alten Jahrgängen deutscher Fischereizeitschriften stöbert, wird feststellen, daß unsere Küstenfischer schon Anfing des Jahrhunderts Probleme mit dem Auftreten von Fischkrankheiten hatten. So wird z. B. berichtet, daß entlang der Elbmiinclung die Flunderfïscher in wirtschaftliche Bedrängnis gerieten, weil ein erheblicher Teil ihres Fanges aufgrund von Infektionen mit der virusbedingten Lymphocystis-Krankheit unvermarktbar war (S c hä percla us [1927]). Und entlang der südwestlichen und südlichen Ostseeküste verendeten während einiger Jahre zur warmen Jahrezeit Aale in Stuickzahlen, die nur noch in Hundertlausenden zu zählen waren (Bruun und Heiberg [1935]; Schäperclaus (1934l). Ursache war, wie wir heute wissen, die bakterielle Vibrio-Krankheit.

Diese Probleme existieren auch heute noch, doch haben sie an Aktualität verloren; denn Flundern werden aufgrund ihres geringen Marktwertes in der Elbmündung heutzutage nicht mehr befischt und die Seuchenzüge beim Ostseeaal verlaufen, vermutlich aufgrund des geschrumptten Bestandes, weniger spektakulär. Bei den Fischkrankheiten haben heute andere Aspekte an Aktualität gewonnen - teils, weil wir neue Kenntnisse gewonnen haben - teils, weil sich unsere Sichtweise geändert hat.

Schließen wir einmal die durch tierische Parasiten verursachten Krankheiten aus, so können wir heute bei den Fischen des Wattenmeeres und der deutschen Ostseekuiste etwa eineinhalb Dutzend Krankheitsformen unterscheiden (Tab. 1). Viele davon sind uns erst seit kurzem bekannt ( $A$ nders und Mölle r \{1992\}). Drei Krankheitstormen, die erst Mitte oder Ende der 1980er Jahre entdeckt wurden, sollen hier kurz vorgestellt werden:

1) Die Maulgranulomatose des Stints ist die häufigste aller Fischkrankheiten im Gebiet Im Wattenmeer sind im Mittel 30\% aller adulten Stinte befallen (A nders und Möller [1991]). Regionaler Schwerpunkt im Auftreten ist die Elbmündung. Symptome sind silberne. halbperlförmige Wucherungen bis zu $8 \mathrm{~mm}$ Durchmesser im Maul- und Kiemenbereich. Hervorgerufen werden sie durch Stacheln, die beim Fressen von Krebsen in die Haut eindringen und dort abbrechen. Um den Fremdkörper herum wuchert dann granulomatöses Gewebe. Die Stacheln lassen sich jedoch nur in sehr jungen Granulomen nachweisen: Später werden sie zersetzt und resorbiert. Dennoch wachsen viele der Granulome weiter - wie wir vermuten, unter dem Einfluß von Pilzen, die eine Beziehung zu Abwassereinleitungen haben könnten (Anders und Möller [1987]). 
Tabelle 1

Regelmäßig in deutschen Küstengewässcrn auftretende Fischkrankheiten.

\begin{tabular}{|c|c|c|}
\hline Name & Ursache & Wirtsfische \\
\hline $\begin{array}{l}\text { Skelettdeformationen } \\
\text { Wirbelsäulenverkürzung } \\
\text { Wirbelsäulenverkrüimmung } \\
\text { Kiefermißbildung } \\
\text { Kiemendeckelverküirzung }\end{array}$ & $\begin{array}{l}\text { im Experiment } \\
\text { zahlreiche Streß- } \\
\text { parameter als } \\
\text { Auslöser }\end{array}$ & $\begin{array}{l}\text { Zahlreiche } \\
\text { Arten }\end{array}$ \\
\hline $\begin{array}{l}\text { Tumoren } \\
\text { Blumenkohlkrankheit } \\
\text { Laichpapillomatose } \\
\text { Papillomatose } \\
\text { Fibromatose }\end{array}$ & $\begin{array}{l}\text { unbekannt } \\
\text { Herpesvirus? } \\
\text { unbekannt } \\
\text { Lentivirus? }\end{array}$ & $\begin{array}{l}\text { Aal } \\
\text { Stint } \\
\text { Kliesche } \\
\text { Steinpicker }\end{array}$ \\
\hline $\begin{array}{l}\text { Tumorähnliche Krankheiten } \\
\text { Lymphocystis } \\
\text { Amöben-Pseudotumor } \\
\text { Maulgranulomatose }\end{array}$ & $\begin{array}{l}\text { Iridovirus } \\
\text { Amöben } \\
\text { Nahrungspartikel, Pilze }\end{array}$ & $\begin{array}{l}\text { Plattfïsche } \\
\text { Gadiden } \\
\text { Stint }\end{array}$ \\
\hline $\begin{array}{l}\text { Geschwürkrankheiten } \\
\text { Vibriose } \\
\text { Flossentäule } \\
\text { Hautnekrose } \\
\text { Gelbe Pest } \\
\text { Kieferentzündung }\end{array}$ & $\begin{array}{l}\text { Vibrio-Bakterien } \\
\text { Bakterien } \\
\text { Fangschäden? } \\
\text { Flexibacter-Bakterien? } \\
\text { unbekannt }\end{array}$ & $\begin{array}{l}\text { zahlreiche Arten } \\
\text { zahlreiche Arten } \\
\text { Plattische } \\
\text { Kabeljau } \\
\text { zahlreiche Arten }\end{array}$ \\
\hline
\end{tabular}

2) Die Gelbe Pest des Kabeljaus wurde zum ersten Mal im Frühjahr 1988 im Wattenmeer nachgewiesen. Da die Krankheitssymtome sehr auffällig sind, aber keine Nachweise aus früheren Jahren oder anderen Gebieten existieren, könnte es sich hier tatsächlich um eine ..neue" Krankheit handeln. Frühstadien sind lïngliche gelbe Cysten in der Haut des Mauls und am Flossenansatz. In einem späteren Entwicklungsstadium löst sich das umgebende Mluskelgewebe auf. Meist scheint diese Krankheit tödlich zu verlaufen. Befallen werden fast ausschließlich Kabeljaue der jüngsten Altersgruppe. Regionaler Schwerpunkt im Auftreten sind die Mündungen von Elbe, Eider. Weser und Schelde. Die höchste bislang ermittelte Befallsrate betrug 15\% im Februar 1988 in der Elbmündung. Ursache für die Gelbe Pest ist vermutlich eine Infektion mit Flexibacter-Bakterien. Der experimentelle Beweis für diese Vermutung steht jedoch noch aus (Hilger et al. [1991]).

3) Die dritte Krankeit befällt den Steinpicker. Die auffälligen gelben Wucherungen traten 1988 und 1989 bei $0.3 \%$ der adulten Fische im Wattenmeer auf. Regionale und saisonale Schwerpunkte im Auftreten waren. vermutlich aufgrund der generell niedrigen Befallsrate, nicht auszumachen. Im April 1992 wurden jedoch gleich mehrere erkrankte Fische in der Eidermündung gefangen. Die Krankheitsursache ist unbekannt. Im elektronenmikroskopischen Bild zeigen sich in dem Tumorgewebe regelmäßig zahlreiche Partikel. die wir vorläufig zu den Lentiviren stellen. Von der Form her ähneln sie dem HIV, dem AIDS-Erreger beim Mlenschen. Von wissenschaftlicher Bedeutung ist hier, daß Lentiviren bislang noch niemals in Tumoren und noch nie in Meerestieren nachgewiesen worden sind (A nders et al. [1991]).

Die Bestandsaufnahme der äußerlich sichtbaren Fischkrankheiten in unseren Küstengewässern ist heute weitgehend abgeschlossen (A n ders und M öller [1992]). Künftig werden vermutlich nur noch wenig neue Formen hinzukommen. Auch über die regionalen und saisonalen Fluktuationen liegt umfangreiches Datenmaterial vor. Nächste Schritte müssen jetzt die Ursachenklärung und danach die Analyse der Umweltbeziehungen dieser Krankheiten sein. Erst danach werden wir auch eine zuverlïssige Bewertung der Bedeutung dieser Krankheiten 
für den Menschen geben können. Zur Zeit sind wir bei wichtigen Fragen noch auf Spekulationen angewiesen, die wir aus Felddaten ableiten, da es nur wenig expcrimentelle Arbeitsansätze gibt.

Auffiillig ist, daß Fischkrankheiten im Wattenmeer mit einer größeren Formenvielfalt und viele von ihnen auch mit höheren Befallsraten auftreten als in Vergleichsgewässem ( $\mathrm{n}$ d e r s und M ölle r [1992]). Die uns zugänglichen Vergleichsdaten sind allerdings sehr lïckenhaft. Ähnlich umfangreiche Bestandsaufnahmen unter Einschluß aller am Ort häiufigen Fischarten wurden bislang nur in der suidwestlichen Ostsee (M öl I e r [1975]), in einem japanischen Fluß (Loganathan et al. [1989]) und an einigen tropischen und subtropischen Standorten (Pe terse n et al. [1992] und unpubl. Daten) durchgefüihrt. Neue Erkenntnisse werden jedoch von einem Forschungsprojekt über das Auftreten von Fischkrankheiten in verschiedenen Ästuaren der Nordsee erwartet, welches derzeit im Auftrag des Umweltbundesamtes durchgeführt wird.

Drei mögliche Ursachen lassen sich für ein gehäuftes Auftreten von Fischkrankheiten im Wattenmeer anführen:

I) hohe Schad- und Nährstoff gehalte,

2) ein instabiles Aquaklima, insbesondere im Hinblick auf stark schwankende Temperaturen und Salzgehalte und

3) ein unnatürliches Beute-Räuber-Verhältnis, in dem aufgrund des Wegfanges aller größeren Raubfische die Kranken und Krüppel relativ gute Überlebenschancen haben.

Innerhalb des Wattenmeeres fällt auf, daß die meisten Inf ektionskrankheiten gehäuft in den Mündungsbereichen der Flüsse auftreten (A nders und MI öller [1992]). Besonders auffällig ist dies für die Gelbe Pest des Kabeljaus und für verschiedene Flunderkrankheiten. Am Beispiel der Elbflunder konnte gezeigt werden, daß hohe Befallsraten im Ästuar gekoppelt sind mit schlechtem Ernährungszustand (M öller [1990]). Dieser wiederum ist bedingt durch akuten Nahrungsmangel. Die sowohl kurzfristig im Tidenverlauf als auch mittelfristig im Jahresverlauf stark schwankenden Salzgehalte in der Elbmündung unterbinden dort die Ausbildung größerer Zoobenthonbestände, welche Flunder und Kabeljau als Nahrung dienen könnten (Fiedler [1991]). Eine ähnliche Situation deutet sich auch für die Mündungsgebiete von Eider und Weser an. Diese natürlichen Vorgänge überdecken die Auswikungen anthropogener Streßparameter, insbesondere bereiten sie uns große Probleme, die Auswirkungen erhöhter Schadstoffgehalte in diesen Gebieten auf die Fischgesundheit zu erkennen (A nders und MI öller [1992]).

Die Bedeutung der Fischkrankheiten in unseren Küstengewässern muß aus drei Blickrichtungen betrachtet werden: aus Sicht der Fische, aus Sicht des Umweltmanagements und aus Sicht der Fischwirtschaft.

Aus Sicht der Fische ist von Bedeutung, daß Krankheiten vermutlich eine der häufigsten Todesursachen im Gebiet sind. Das gilt allerdings weniger für Tumor- und Infektionskrankheiten, sondern vor allem für parasitenbedingte Krankheiten. An erster Stelle zu nennen ist hier der Copepode Lernaeocera branchialis, der im Jahresmittel bei etwa 15\% der Kabeljaue und 32\% der Wittlinge im Wattermeer auftritt. Infizierte Fische magern stark ab und sind deutlich anfälliger für Streßsituationen, etwa für Sauerstoffmangel im Wasser ( A n d e rs und M ölle r [1992]; M ölle r [1983]; S cholz [1986]). Vermutlich gehen infizierte Fische mehrheitlich zugrunde. Larven dieses Parasiten leben auf den Kiemenblättern von Flundern, wo sie ebenfalls beträchtliche Schäden hervorrufen (W i c h o w s k i [1990]). Eine Reduzierung der Schwimmleistung wurde für Aale und Stinte nachgewiesen, die mit dem Nematoden Anguillicola crassus bzw. dem Einzeller Pleistophora ladogensis oder dem Nematoden Pseudoterranova decipiens befallen waren (S prengel und Lüchtenberg [199I]). Infizierte Fische werden damit anfälliger für Freßfeinde. Beim Aal ist zudem mit einer verringerten Reproduktionsleistung zu rechnen, wenn infizierte Fische auf grund ihrer Behinderung das Laichgebiet in der Sargasso See nicht mehr erreichen können (M öl le r et al. [199I]). Welche Bedeutung diese Krankheitsformen auf Populationsebene haben, ist unbekannt. 
Sehr viel mehr Aufmerksamkeit wird in Deutschland derzeit der Frage gewidmet, inwieweit das Auftreten von Fischkrankheiten als Indikator füir die Meeresverschmutzung gewertet werden kann. Hinsichtlich der Beweisführung befinden wir uns hier in einem Dilemma. Zum einen wissen wir aus der Human- und Tiermedizin und nicht zuletzt auch aus der Fischzucht, daß Schadstoffe in cler Atemluft bzw. im Wasser und in der Nahrung das Auftreten spezieller Tumorformen und Infektionskrankheiten auslösen oder fördern. Es gibt keinen Grund für die Annahme, daß dieses Prinzip nicht auch bei freilebenden Fischen gelten sollte. Andererseits ist es, zumindest für den Bereich der Nordsee, noch nicht gelungen, überzeugende Beweise dafür zu erbringen. Hierf ür gibt es nur zwei mögliche Erklärungen: Entweder sind unsere bisherigen Forschungsansätze nicht geeignet gewesen, um diese Zusammenhänge nachzuweisen, oder aber die Schadstoffbelastung in der Nordsee ist so gering, daß die Gesundheit der dort lebenden Fische nicht akut get ährdet ist.

Aus Sicht der Fischwirtschaft sollten einige der aktuellen Befunde aus cler Forschung zur Sorge Anlaß geben. Die seit Herbst 1991 wieder in der westlichen Ostsee grassierende Ichthyophonus-Krankheit, die auf einer Infektion mit encloparasitischen Pilzen beruht, hat bereits zu einer spürbaren Beeinträchtigung der heringsverarbeitenden Industrie in Schweden geführt (Thuli n, pers. Mitt.). Eine Krankheit wie die Gelbe Pest könnte schnell zu einem wesentlichen Störfaktor in der sich gerade ent wickelnden Kabel jauzucht in Norwegen werden. Und auch für die deutsche Fischerei, für die der Kabeljau eine wesentliche Einnahmequelle darstellt, ist der Befund nicht ohne Bedeutung. Der Fischkonsum wird sichtlich nicht angeregt, wenn sich herumspricht. claß einigen der Kabel jaue in unseren Küstengewässern der Kopf bei lebendigem Leibe abfault. Sollte sich die Vermutung bestätigen, daß es sich bei den Partikeln in den Steinpickerturmoren um eine HIV-ühnliche Virusform handelt, könnte das Wattenmeer ganz schnell zu dem werden, was sich viele Naturschützer wünschen: zu einem von der Fischerei unbeeintlußten Nationalpark. Steinpicker dienen nicht der menschlichen Ernährung, aber viele dieser Fische werden an Bord der Krabbenkutter zusammen mit den Garnelen gekocht. Und freigesetzte bzw. an Gewebereste gebundene Viren werden u. a. von Muscheln filtriert.

Ob diese neuen Krankheitserreger irgendeine Bedeutung für die menschliche Gesundheit haben, läßt sich nicht beantworten, bevor man die Erreger nicht identifiziert und charakterisiert hat. Das aber ist noch nicht geschehen. Allgemein gilt es als wenig wahrscheinlich, daß Bakterien oder Viren von Fischen auf Menschen übertragen werden. Die derzeit in Südamerika grassierende Cholera und das Calicivirus kalifomischer Küstenfïsche, das bereits zu Mortalitäten in der Schweinezucht vor Ort geführt hat ( $\mathrm{Smith}$ [1980), deuten jedoch an, daß Ausnahmen durchaus möglich sind.

In keinem anderen Land reagiert die Bevölkerung so überzogen auf tatsächliche oder eingebildete Umweltprobleme wie in Deutschland. Algenpest. Seehundsterben, schadstoftbedingte Fischkrankheiten und Würmer im Fisch sind da nur einige Beispiele aus dem Bereich der Marinen Pathologie. Die hier vorgestellten neuen Befunde über Fischkrankheiten in unseren Küstengewässem reichen zwar für eine sichere Bewertung des von ihnen ausgehenden Risikos nicht aus, sie bieten aber allemal genügend Stoff für Berichte in der Presse, die der deutschen Fischwirtschaft wieder Millionenverluste einf ahren könnten. Der Nematodenskanclal des Jahres 1987 ist da sicherlich noch in guter Erinnerung. Auch damals waren weniger Sachverhalte für die Kaufzurüickhaltung der Bevölkerung ausschlaggebend, sondern vor allem die Unsicherheit. mit der zuständige Stellen dem Problem anfangs begegneten.

Auffällig ist für mich, daß deutsche Wissenschaftler in der Vergangenheit zwar bei der Entdeckung und mediengerechten Präsentation solcher Probleme wesentlich beteiligt waren. daß sie aber zur Analyse und Lösung dieser Probleme stets nur bescheidene Beiträge geleistet haben. Es waren niederländische und dänische Fachleute, welche die Ursache des Seehundsterbens entdeckten (Osterhaus et al. [1988]). Japanische, kanadische und niederländische Wissenschaftler haben die wichtigsten Beiträge zur Aufdeckung und Lösung des Nematodenproblems im Fisch geliefert (Bow en [1990]; I s h i k u r a and Kikuchi [1990]; Th ie I et al. [1960]) und amerikanische und schwedische Wissenschaftler waren es, die bislang als einzige 
überzeugend Zusammenhänge zwischen Gewässerverschmutzung und dem Auftreten von Fischkrankheiten aufzeigen konnten (Malins et al. [19S5]; Lindesjöö and Thulin (1990]).

Die Ursache hierfïir sehe ich in einer zunehmenden Abwendung von der kontinuierlichen und wissenschaflich fundierten Forschung hin zum Datensammeln und zur Feuerwehrforschung in unserem Land. Eine ganze Reihe von Feldforschungsprojekten der letzten Jahre hat uns mittlerweile gewaltige Datenberge beschert. Diese sind zwar dazu geeignet. Zustandsbeschreibungen zu geben und rechnerische Korrelationen zwischen abiotischen und biotischen Parametern aufzuzeigen. Sie allein reichen jedoch nicht aus, um biologische Reaktionen zu erklären oder gar vorherzusagen. Feuerwehrprojekte laufen bei uns immer nach Bekanntwerden neuer Umwelt"katastrophen" an. Häufig werden mit solchen Projekten dann Wissenschaftler beauftragt. die zwar renommiert sind. denen es aber an speziellem Grundlagenwissen mangelt, um diese Probleme auch schnell in den Griff zu bekommen. Bei einigen Wissenschaftlern zeigt sich zudem eine Tendenz hin zu der Einstellung: Wir haben keine Zeit mehr zum Forschen - wir müssen handeln. Dies ist der direkte Weg vom überlegten Handeln hin zum Aktionismus. Sicherlich hat in bestimmten Situationen auch der Aktionismus seine Berechtigung, man sollte ihn aber den dafür besser geeigneten Gruppen unserer Gesellschaft überlassen.

Ich neide keinem Kollegen seine Datensammelprojekte, für die Zukunft sehe ich aber keine großartigen Erfolgsmöglichkeiten, wenn nicht der zielgerichteten Grundlagenforschung wieder ein gebührend breiter Raum zugestanden wird. Für das Gebiet der Marinen Pathologie bedeutet dies vor allem eine Stärkung experimenteller, sowie biochemisch- und mikroskopisch-diagnostischer Arbeitsansïtze. Künftig auftretende Probleme werden wir nur dann rechtzeitg bewerten und lösen können. wenn wir auf ein ausreichend breites und fundiertes Grundlagenwissen zurückgreifen können. So etwas füllt jedoch nicht vom Himmel. Es muß langtristig geplant und kontinuierlich entwickelt werden. Und hier gilt es, Versäumtes nachzuholen.

\section{Literatur}

Anders, K., I. Hilger and H. Möller, 1991: Lentivirus-like particles in connective tissue tumors of fish from German coastal waters. Dis. aquat. Org. 11, $151-154$.

A nders. K. and H. Mäller, 1987: Food- induced granulomatosis in European smelt. Osmerus eperlanus. Can. J. Fish. aquat. Sci. 44. 1848-1854.

A nders, K. und H. Möl!er, 1991: Epidemiologische Untersuchungen von Fischkrankheiten im Wattenmeer. Ber. Inst. Meeresk. Kiel 207. $166 \mathrm{~S}$

Anders, K. und H. Möller, 1992: Atlas der Fischkrankheiten im Wattenmeer/Atlas of fish diseases in the Wadden Sea. Berlin: E. Schmidt Verlag, $112 \mathrm{~S}$.

B ow e n. W. D. (ed.), 1990: Population biology of sealworm (Pseudoterranova decipiens) in relation to its intermedeiate and seal hosts. Can. Bull. Fish. aquat. Sci. 222, 1-306.

Bruun, A. F. und B. Heiberg, 1935: Weitere Untersuchungen über die Rotseuche des Aales in dänischen Küstengewässern. Z. Fisch. 33 , 379-382.
Fiedler, M., 1991: Die Bedeutung von Makrozoobenthos und Zooplankton der Unterelbe als Fischnahrung. Ber. Inst. Meeresk. Kiel 204, $226 \mathrm{~S}$.

Hilger. I..S. Ullrich and K. Anders, 1991: A new ulceruive flexibacteriosis-like disease ("yellow pest") affecting young cod Cradus morhua from the German Wadclen Sea. Dis. aquat. Org. 11. 19-29.

Ishikura. H. and K. Kikuchi (eds), 1990: Intestinal anisakiasis in Japan. Tokyo: Springer $265 \mathrm{pp}$.

Lindesjöö. E. and J. Thulin, 1990: Fin erosion of perch Perca fluviatilis and ruffe Gymnocephalus cernua in a pulp mill effluent area. Dis. aquat. Org. 8, 119-126.

Loganathan, B. G.. S. Tanabe, R. Tatsuk aw a, K. Og awa, M. Goto, 1989: Temporal changes of morphologic abnormalities and parasitic infestation in fish from the River Nagaragawa. Japan. Nipp. Suis. Gakk. 55. 769-774.

M alins. D. C., D. D. McCain. M. M. Krahn, M. S. Myers. J. E. Stein, W. T. Roubal. D. W. Brown. U. Varanasi. H. O. Hodgins, and S. L. Chan, 1985: Occurrence 
of hepatic neoplasms and other lesions in bottom-dwelling fish and relationship to pollution in Puget Sound, Washington. In: Jolley, V. A.. R. J. Bull. IV. P. Davis, S. Kiltz, M. H. Roberts and V. A. Jacobs (eds): "IVater chlorination: chemistry. impact and heilth elfects", Vol. 5, 399-41t. Chelsea. Michigan: Lewis Publishers.

Möller. H., 1975: Der Einfluß von Temperalu und Salzgehalt aut Entwichlung und Verbreitung von Fischparasiten. Dissertation, math.-nat. Fak. Univ. Kiel, I08 S.

Möller. H. . 1983: The effects of Letnacocera-infestation on cod (Gaddus morhuil). Bull. Eur. Ass. Fish Pathol. 3, 21-22.

Miller, H., 199(): Association between disealses of flounder (Platichthys hesus) and environmental conditions in the Elbe estuary, FRG. J. Cons. int. Explor. Ner 46, 187-199.

Nöller, H. and K. Anders. 1992: Epidemiolog' of fish cliseases in the Wacklen Sea. ICES J. mir. Sci. 49, 199-208.

Möller. H.. S. Holst. H. Lü chtenberg and F. Petersen, 1991: Infection of eel Anguilla anguilla from the River Elbe estuary with iwo nematodes. Anguillicola crassus and Pseudoterranova decipiens. Dis. aquat. Org. 11. 193-199.

Osterhaus. A. D.. J. Groen. P. de Vires. and F. G. Uytdeh a g, 1988: Canine distemper virus in seals. Nature $335,4() 3-4() 4$
Petersen. F., H. Palm, H. Möller and M. A. Cusi. 1992: Muscle parasites of fish from central Philippine waters. Acyuat. L.iv. Res. (in pressi).

Schäpercla us, W.. 1927: Lymphocystisertiankungen bei Flundern und Schollen und ihre Bchimptung. Mitc. de. SeetischVer. 43, 96-99.

Schäperclat us. W.. 1934: Untersuchungen über die Ailseuchen in deutschen Binnen- und Kiistengewiissern. Z. Fisch. 32. 191-217.

Scholz, U. 1986: Siluerstoffmangelresistenz von Küstenfischen unter dem Eintluß3 von Parsitierung, Diplomarbeit, math.-natt. Fatk. Univ. Kiel, $65 \mathrm{~S}$.

Smith. A. W.. D. E. Skilling. A. H. Dardiri and A. B. Lathatm, 19S0): Callicivirus pathogenic for swine: a new serotype isolated from opaleye Girella nigricans, an ocean rish. Science, N. Y. 2()9. 94()-941.

Sprengel, G. and H. Lüchtenberg. 1991 Infection by endoparasites reduces maximum swimming speed of European smelt Osmerus eperlanus and European eel Anguilla anguilla. Dis. aquat. Org. 11, 31-35.

Thiel, P. H. van, F. C. Kuipers and R. T. Roskam. 1960: A nematode parasitic to herring, causing acute abdominal syndromes in màn. Trop. Geogr. Med. 12. 97-113.

Wichowski. F. J., 1990: Parasiten als Inclikator von Wanderungen der Elbrlunder Platichthys thesus (Linnileus, 1758). Fischökologie 2, 1-26.

Anschrift des Verfassers:

Prof. Dr. H. Möller

Institut für Meereskunde an der Universität Kiel

Düsternbrooker Weg 20

2300 Kiel 1 